\title{
Dual Sized Varying Slot Lengths Loading Dielectric Resonator Reflectarray
}

\author{
A. M. Abd-Elhady ${ }^{1, *}$, S.H. Zainud-Deen ${ }^{2}$, A. A. Mitkees ${ }^{3}$, A. A. Kishk ${ }^{4}$ \\ ${ }^{1}$ Faculty of Engineering, Benha University, Egypt \\ ${ }^{2}$ Faculty of Electronic Engineering, Monofia University, Egypt \\ ${ }^{3}$ Military Technical College, Egypt \\ ${ }^{4}$ Department of Electrical Engineering, Concordia University, Canada
}

\begin{abstract}
A reflectarray antenna consists of elements of rectangular dielectric resonator (DRA) with slot loading of different lengths is proposed for bandwidth enhancement. Two DRA sizes an two slot widths are available to tune the phase of each element in the reflectarray so that a full 360 degrees phase shifts can be achieved by superposition. Two structures are presented in that paper. The first is center fed reflectarray while the second is offset fed for decreasing the feeder blockage. The antenna has $10 \%$ bandwidth for $1 \mathrm{~dB}$ gain variation is obviously wider than that of conventional reflectarray antenna while the offset fed reflectarray provide better far field pattern with back lobes reduction by $-5 \mathrm{~dB}$ and side lobe by $-2 \mathrm{~dB}$. A pyramidal X-band horn was used in both reflectarrays which have $23 \times 23$ elements of with cells separation of 12 $\mathrm{mm}$ that less than $15 \mathrm{~mm}$ (lambda/2) for avoiding grating lobes. The analyses are carried out using the finite integration technique (FIT) and the transmission line method (TLM) with good agreements between them.
\end{abstract}

Keywords DRA, Slot loaded DRA, Reflectarray, Planar array

\section{Introduction}

High gain antennas are desired in various applications, such as radar, radio astronomy, satellite communications, and remote sensing. The conventional parabolic reflectors are generally bulky in size and large in mass due to the curved reflecting surfaces. Recently, microstrip reflectarrays are found to be very attractive aperture antennas because of their planar structure and simple feeding[1]. Its advantage over a conventional planar microstrip phased array is a low conduction loss, especially in a millimeter-wave region[2,3], because it uses a spatialtype beamforming network. However, its shortcoming is a narrow-band operation compared with the conventional parabolic reflector. Usually, the required phase value to compensate for the different paths of the wave from the feed horn to each array element is only achieved at the center frequency within a range of $360^{\circ}$. Each element phase can be adjusted to produce a required phase over the aperture and control the main beam orientation. Usually, the dynamic range of the phase shift in a single-layer reflectarray is less than $360^{\circ}$, and the complete compensation is only for central frequency, which restrict the application to the large-aperture and/or wideband reflect-array. The Problem of narrow-band operation of

* Corresponding author:

abdoeng78@gmail.com (A. M. Abd-Elhady)

Published online at http://journal.sapub.org/ijea

Copyright (C) 2012 Scientific \& Academic Publishing. All Rights Reserved single layer patch reflectarrays can be overcome by employing multi-layer variable size patches[4-5]. The use of stack patches improves the phasing range of the individual elements (to about $450^{\circ}$ for two layers) by using dual patch sizes, the upper bigger patch is resonated for the lower frequency while the smaller patch for the upper frequency. That technique broaden the bandwidth by superposition the two bandwidths. That offers lower phase slopes and leads to an increased operational bandwidth of the entire array antenna. A new dual layer cell is designed with wide phase range than dual layer patch reflectarray[6]. Another way for extending the bandwidth of the reflectarray, a patch supported on variable slot length is employed as the element of reflectarray, where the length of slot determines the reflection phase from the element for compensating the ray-path difference from the feed[7-11]. Overcoming the feed blockage that degrade the radiation efficiency of antennas as blocking by horn feeder some of reflected field, An offset reflectarray was designed using variable patch sizes[12]. The mutual coupling between microstrip elements printed on standard substrates becomes significant; in addition, the conductor and surface wave loss are severe. To overcome these limitations, other candidate, dielectric resonator antennas (DRA) have been introduced due to their low loss, relatively wide bandwidth, high radiation efficiency and low mutual coupling. These reflectarray antennas realized by rectangular and crossed dielectric resonator for linear polarization[13] which supported by strip for gain improvement while a Ka-band variable DRA 
reflectarray lengths supported on substrate of same dielectric constant of DRA was designed[14]. A X-band linear and circular polarized DRA are investigated[15]. A X-band Linear polarized reflectarray supported on slot with variable lengths was investigated to obtain a complete period phase cycle[16]. Aperture coupled DRA with variable strip line at X-band was design with good performance in radiation patterns and total efficiency[17]. In this paper, DRA reflectarray with slot-loading is investigated. The phase of reflection coefficient is adjusted by tuning the length of the slot. However, two DRA sizes and two slot widths are used. These two parameters (the slot width and DRA sizes) are available to tune the phase of each element in the reflectarray so that a full 360 degrees phase shifts can be achieved. The analyses are carried out using the finite integration technique (FIT)[18] and the transmission line method (TLM)[19].

\section{Simulation Analysis}

A DRA reflectarray with variable slot loading length supported on dielectric substrate with conducting ground plane is constructed as shown in Figure 1. The unit cell consists of rectangular DRA, with length $L$, width $W$, height $H$, a slot in the ground plane, with length $L_{s}$ and width $W_{s}$, a dielectric layer of thickness $h$, and a conducting ground plane. The bottom conducting plate is acting as a reflector to reduce the back radiation and improve the front to back ratio of the main beam. In order to determine the relation between the reflected phase and the selected parameters, a unit cell in an infinite periodic structure is considered with normal plane wave incidence. The design center frequency is considered $10 \mathrm{GHz}$. Figure 2 shows the phase variations versus the selected parameters. Two behaviours can be observed in Figure 2a: for $0<L_{s}<5 \mathrm{~mm}$ with $L=W=7$ $\mathrm{mm}$ and $W_{s}=1.4 \mathrm{~mm}$, a $240^{\circ}$ phase range can be obtained; for $5<L_{s}<11 \mathrm{~mm}$ with $L=W=10 \mathrm{~mm}$, another $120^{\circ}$ phase range is reached. The complete phase $360^{\circ}$ is obtained by superposition as shown in Figure 2b. FIT method (present method), the transmission line (TLM) method and commercial HFSS software[20] are used to investigate the performance of the antenna for the variation of the phase of the reflection coefficient against the slot length. The phase of the reflection coefficient for different frequencies as a function of DRA element length is computed. The phase of reflection coefficients as a function of the plane wave angle of incidence is also predicted. The phase variation due to the angle of the incidence plane wave between normal incidence and $30^{\circ}$ is found to be less than $90^{\circ}$ as illustrated in Figure 3. Therefore; phases of the reflection coefficient due to normal plane wave incidence on a periodic infinite array are used to design the reflectarray and found to be sufficient as will be seen later. Considering the array on the $\mathrm{x}-\mathrm{y}$ plane illuminated by a feed horn, the required phase distribution, $\varphi\left(x_{i}, y_{i}\right)$, at each element of the array to collimate a beam in the $\left(\theta_{o}, \varphi_{o}\right)$ direction is determined as :

$$
\begin{aligned}
& \varphi\left(x_{i}, y_{i}\right)=k_{0}\left(d_{i}-\sin \theta_{0}\left(x_{i} \cos \phi_{0}+y_{i} \sin \phi_{0}\right)\right] \\
& d_{i}=\sqrt{\left(\left(x_{c e}-x_{f 0}\right)^{2}+\left(y_{c e}-y_{f 0}\right)^{2}+\left(z_{f 0}\right)^{2}\right)}
\end{aligned}
$$

where $k_{o}$ is the propagation constant in vacuum, $d_{i}$ is the distance from the feed horn phase center to the element $i$ of the array and $\left(x_{i}, y_{i}\right)$ are the coordinates of the center of element $i$. Getting the full phase of $360^{\circ}$, two DRA elements and two slot widths are used. First DRA has dimension of square $(L=W=7 \mathrm{~mm})$ and slot width $W_{s}=1.4 \mathrm{~mm}$, while the second has dimension of square $(L=W=10 \mathrm{~mm})$, and slot width $W_{s}=0.7 \mathrm{~mm}$. The DRAs have height $H=3 \mathrm{~mm}$ with relative dielectric constant $\varepsilon_{\mathrm{r}}=10.2$. The dielectric layer thickness is $2 \mathrm{~mm}$ with $\varepsilon_{\mathrm{r}}=1$. Variable slot length $l_{s}$ is used.

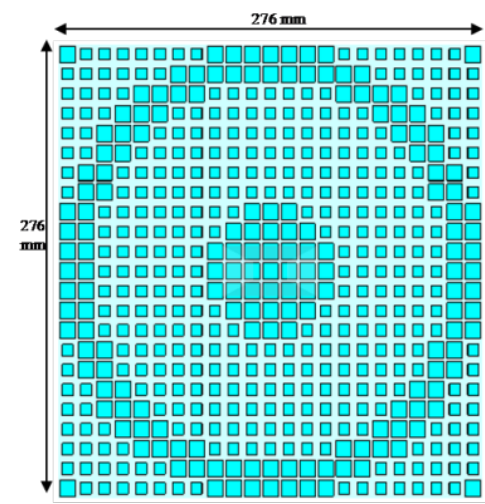

(a)

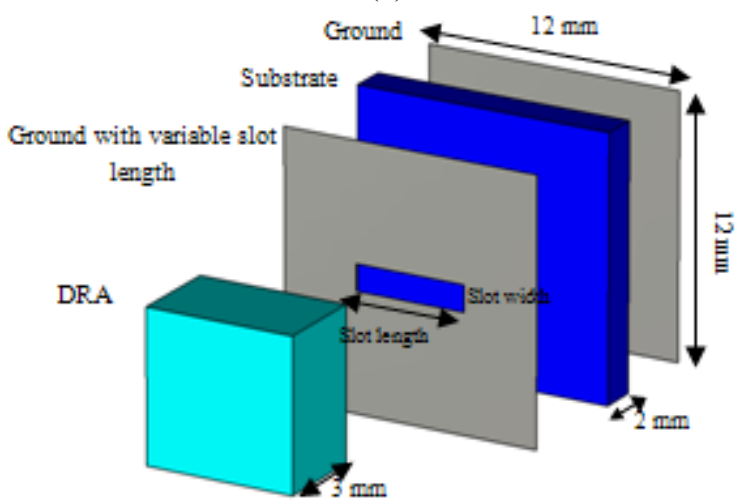

(b)

Figure 1. DRA layout reflectarray a) Top array view b) Cell side view.

\section{Center Fed Reflectarray}

The feed position is at $x_{f o}=0 \mathrm{~mm}, y_{f o}=0 \mathrm{~mm}$ and $z_{f o}=$ $345 \mathrm{~mm}$, with respect to the reflectarray center. The array is 23 x 23 unit cells. The reflectarray size was $276 \times 276 \mathrm{~mm}^{2}$ with elements spacing of $12 \mathrm{~mm}$ and the focal length-todiameter ratio $\mathrm{F} / \mathrm{D}=1$. The layout of the DRA elements and their relative sizes are shown in Figure 1. A pyramidal horn of dimensions $60 \times 30 \mathrm{~mm}^{2}$ is used. The radiation patterns of the designed reflectarray at $10 \mathrm{GHz}$ are shown in Figure 4. The accuracy of the radiation patterns are verified by comparing the results of the present method with those from the TLM method. 


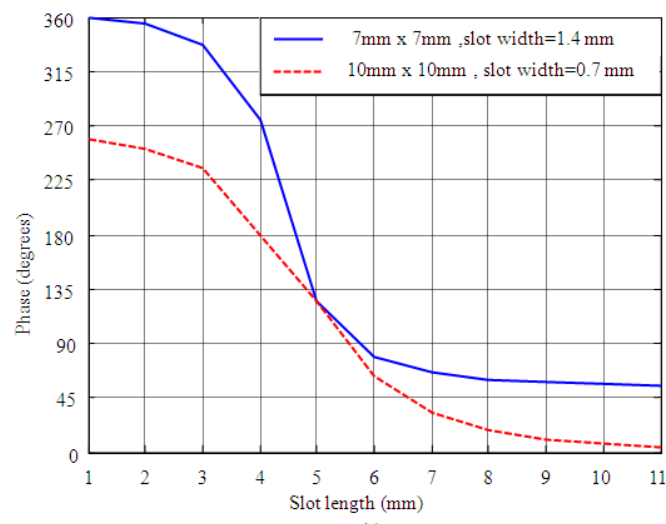

(a)

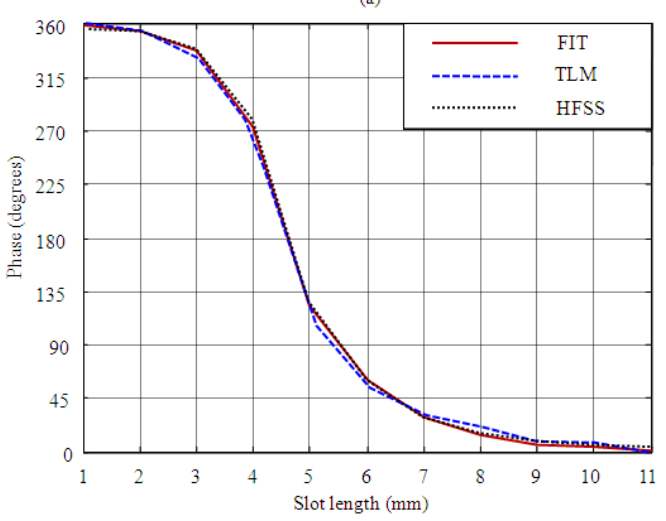

(b)

Figure 2. Reflection coefficient phase versus DRA with variable slot length a)Super position b) Comparison.

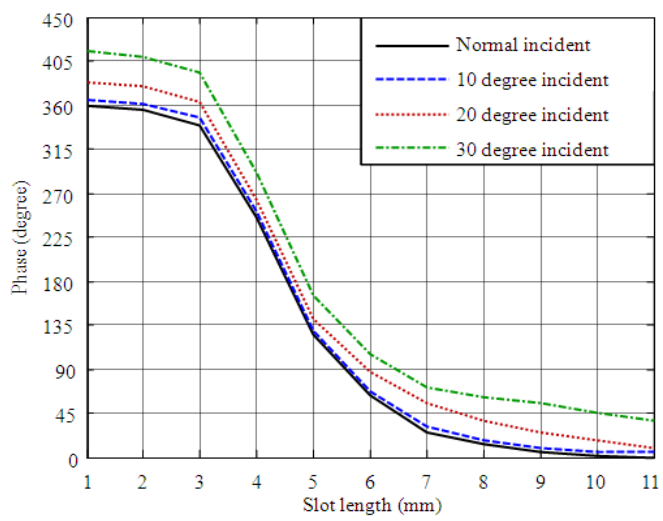

Figure 3. Reflection coefficient phase versus variable length for different oblique angles

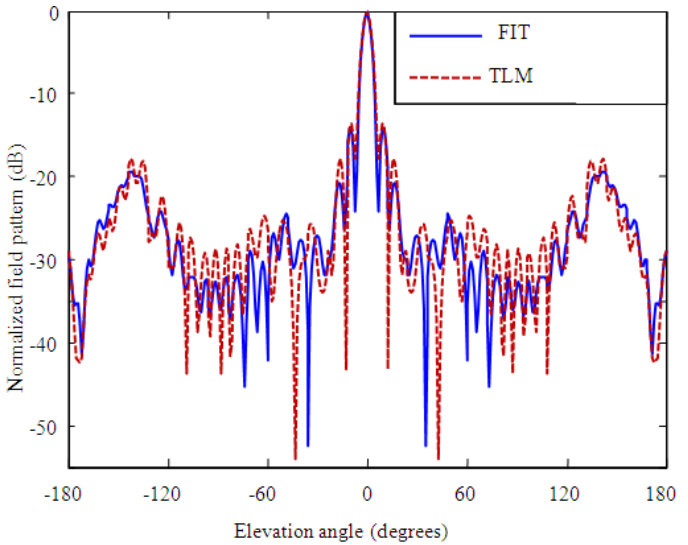

(a)

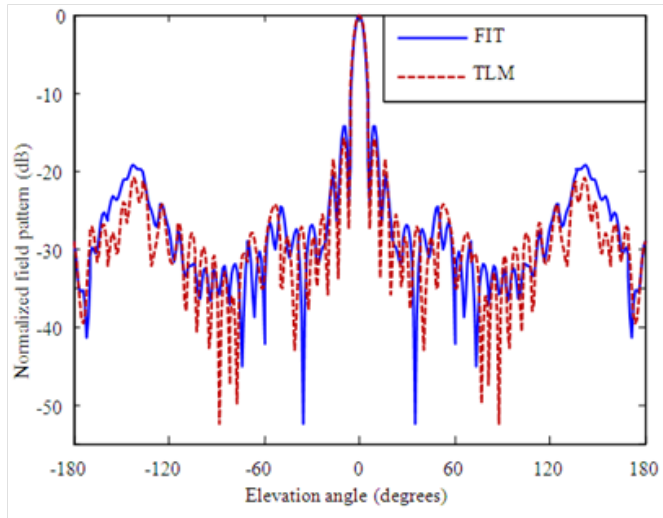

(b)

Figure 4. Reflected Field patterns of DRA reflectarray at $10 \mathrm{GHz}$ at $\theta=$ 0o a) XZ plane( H plane ) b) YZ plane( E plane )

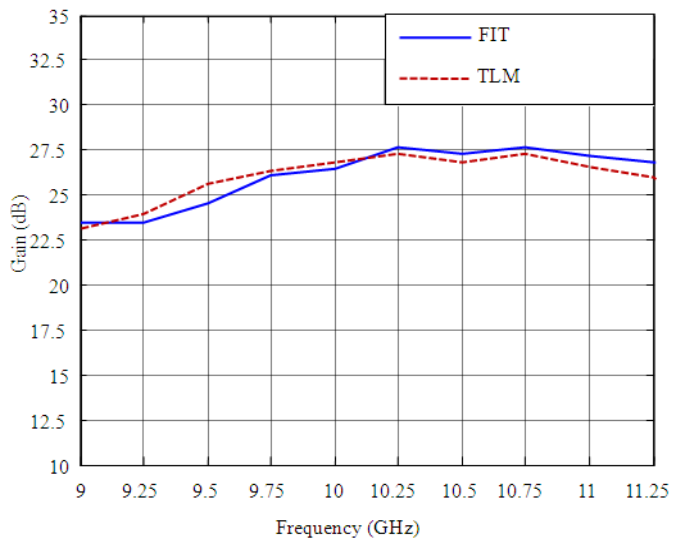

Figure 5. Gain versus frequency

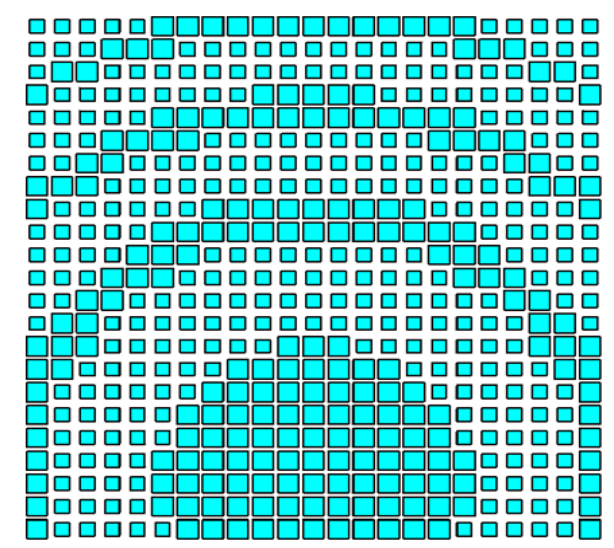

Figure 6. Offset fed DRA layout reflectarray.

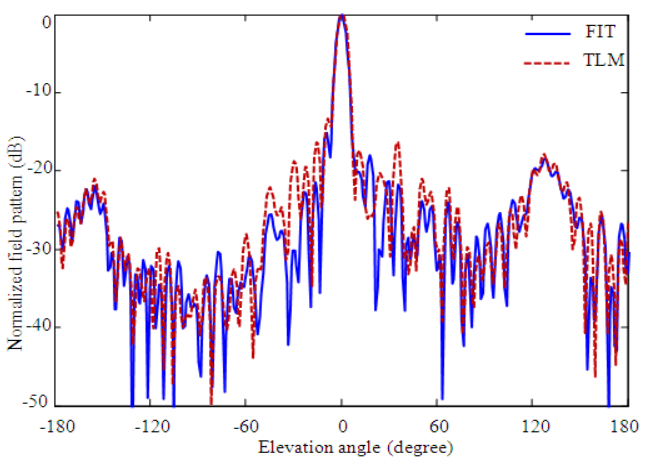

(a) 


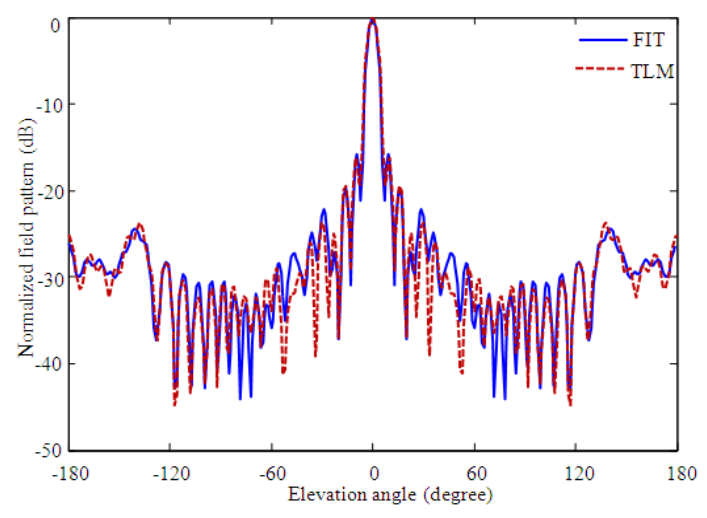

(b)

Figure 7. Reflected Field patterns of DRA reflectarray at $10 \mathrm{GHz}$ at $\theta=$ $0^{\circ}$. a) YZ plane( E plane ) b) XZ plane( $\mathrm{H}$ plane )

The radiation patterns has a $3-\mathrm{dB}$ beamwidth of $8^{\circ}$ and a peak gain of $26 \mathrm{~dB}$ at $\theta=0^{\circ}$. The antenna gain versus frequency is shown in Figure 5 indicating $10 \%$ bandwidth with gain variations of $1 \mathrm{~dB}$.

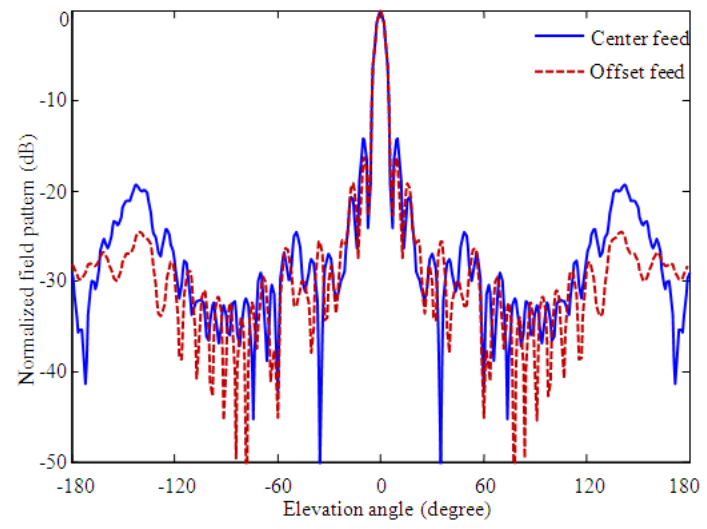

Figure 8. XZ plane( $\mathrm{H}$ plane ) reflected Field patterns comparison

\section{Offset Fed Reflectarray}

According to the center fed reflectarray, the horn block some of reflected field and as a result, the total efficiency of reflectarray was degraded. An offset fed reflectarray was designed to minimize the feeding blockage.

The $\mathrm{H}$ plane comparison between center fed and offset feed is illustrated in Figure 8 . The back lobes is being better in offset by $-5 \mathrm{~dB}$ with side lobe decreasing by $-2 \mathrm{~dB}$.

The accuracy of the radiation patterns are verified by comparing the results of the present method with those from the TLM method.

\section{Conclusions}

Slot-loading rectangular DRA elements reflectarray antenna was designed for linear polarization at X-band. The antenna was simulated by two feeding ways, one is center while the second is offset fed. Both arrays was fed by linearly polarized pyramidal horn.

The cell reflection phase complete period was obtained by superposition of two DRA sizes with variable slot lengths. The array cells were $23 \times 23$ elements reflectarray with area of $276 \times 276 \mathrm{~mm}^{2}$. The finite integration technique was used to compute the radiation patterns and the gain of the antenna. The method was verified by comparing the results with transmission line method and was found to be efficient and accurate for these analyses. The offset fed reflectarrayprovided better performance in reflected field patterns in back lobes and side lobes.

\section{REFERENCES}

[1] J. Huang and J.A. Encinar, Reflectarray antennas, John Wiley \& Sons, Inc., Publication, 2008.

[2] Pozar, D. M., S. D. Targonski, and H. D. Syrigos, "Design of millimeter wave microstrip reflectarrays", IEEE Transactions on Antennas and Propagation, Vol. 45, No. 2, 287-296, Feb. 1997.

[3] F.-C. E. Tsai and M. E. Bialkowski, "Designing a 161element $\mathrm{Ku}$-band microstrip reflectarray of variable size patches using an equivalent unit cell waveguide approach", IEEE Trans. Antennas and Prop. vol. 51, No. 10, pp. 29532962, Oct. 2003.

[4] Encinar, J. A., "Design of two-layer printed reflectarrays for bandwidth enhancement", IEEE 1999 Antenna Propagat. Symp. Dig., pp.1164-1167, 1999.

[5] Encinar, J. A., "Broadband design of three-layer printed reflectarrays", IEEE Transactions on Antennas and Propagation, Vol. 51, No. 7, pp.1662-1664, July 2003.

[6] Wahid, A.; Rahim, M.K.A.; Zubir, F.;" Analysis of dual layer unit cell with minkowski radiating shape for reflectarray antenna on different substrate properties", Applied Electromagnetics (APACE), 2010 IEEE AsiaPacific Conference on 9-11, pp.1-5, Nov. 2010.

[7] M.R.Chaharmir, J.Shaker, M.Cuhaci and A.Sebak; "Reflectarray with variable slots on ground plane" IEE Proc. - Microwave Antennas Propag., Vol.150,No.6,pp. 436 - 439, Dec. 2003.

[8] Marek E.Bialkowski and Feng-Chi E. Tsai "A unit cell waveguide model of a reflectarray formed by microstrip patches and slots" Microwave and Optical Technology Letters, Vol.36, No.3,pp.206-210, Feb. 5, 2003.

[9] D.Cadoret, A.Laisne, R.Gillard, and H.Legay; "A new reflectarray cell using microstrip patches loaded with slots "Microwave and optical technology letters,Vol.44, No.3,pp.270-272, Feb. 5, 2005.

[10] D. Cadoret, A.Laisne, R.Gillard, L.Le Coq and H.Legay "Design and measurement of new reflectarray antenna using microstrip patches loaded with slot" Electronic Letters 26th May, 2005, Vol.41, No.11,pp. 623 - 624.

[11] The-Nan Chang and Chia-Hsin Chung ;"Two-layer Variable Slot Length Reflectarray " PIERS, Hangzhou, China,pp.189191, Mar. 24-28, 2008.

[12] R.D. Javar, X.-D. Wu, and K. Chang, "Offset-feed micro 
strip reflectarray antenna," Electron. Lett., vol.30, no.17, pp.1363-1365, Aug. 1994.

[13] M. H. Jamaluddin, R. Gillard, R. Sauleau, P. Dumon and L. Le Coq, "Reflectarray element based on strip-loaded dielectric resonator antenna", Electron. Lett., vol. 44, No. 11, pp. 664-665,May 2008.

[14] M. G. Keller, J. Shaker and A. Petosa, A. Ittipiboon, M. Cuhaci, Y. M. M. Antar, "A Ka-band dielectric resonator antenna reflectarray," European Microwave Conference 2000, Paris, France, pp. 272-275, Oct. 2000.

[15] S.H. Zainud-Deen, Abd-Elhady, A.A. Mitkees and A.A. Kishk, "Design of Dielectric Resonator Reflectarray Using Full-Wave Analysis," 26th National Radio Science Conference (NRSC 2009), Faculty of Engineering, Future Univ., Egypt, B10, pp. 1-9, Mar. 2009.

[16] M. Abd-Elhady, S.H. Zainud-Deen, A.A. Mitkees and A.A. Kishk, "Slot-Loading Rectangular Dielectric Resonator
Elements Reflectarray“,1st Middle East Conference on Antennas and Propagation, (MECAP), Cairo, Egypt, pp.1-3, Oct. 2010.

[17] A.M. Abd-Elhady, S.H. Zainud-Deen, A.A. Mitkees Ahmed A. Kishk, " X-Band Linear Polarized Aperture-Coupled DRA Reflectarray," 2010 International Conference on Microwave and Millimeter Wave Technology, Chengdu, China, pp. 1042 - $1044,2010$.

[18] M. Clemens and T. Weilande, "Discrete electromagnetism with the finite integration technique," Progress in Electromagnetics Research, PIER 32, pp.65-87, 2001.

[19] C. Christopoulos, The transmission-line modeling method, TLM, IEEE Press, Piscataway, USA, 1995.

[20] HFSS v. 11 ,www.ansoft.com 